\title{
The DC field components of horizontal and vertical electric dipole sources immersed in three-layered stratified media
}

\author{
D. Llanwyn Jones, C. P. Burke \\ Physics Department, King's College, Strand, London, WC2R 2LS
}

Received: 24 September 1996 / Accepted: 3 December 1996

\begin{abstract}
Formulas for computing the Cartesian components of the static (DC) fields of horizontal electric dipoles ( HEDs) and vertical electric dipoles ( VEDs) located in the central zone of a three-layer horizontally stratified medium are derived and presented in a summary form suitable for immediate computation. Formulas are given for the electric and magnetic field components in the upper and central regions. In the general case the computation involves the summation of a convergent infinite series. For the particular case of an infinitely thick central region (corresponding to the twolayer problem), the analysis produces relatively simple closed-form equations for the field components which are suitable for a 'hand calculation'. Specimen calculations for dipoles in seawaters are included and the derived results are compared with computations made using an ac model.
\end{abstract}

\section{Introduction}

The subject of the electromagnetic fields excited by buried or submerged radiation sources is of interest both for remote sensing and for communication purposes. Current elements or probes with various configurations may be utilised as sources. Additionally, it is known that earthquakes generate a characteristic precursor at ultralow (and higher) frequencies (e.g. Molchanov et al., 1992; Parrot et al., 1995) and the formulas presented here may be of value in estimating the source magnitude from measurement of ULF fields on the Earth's surface.

A seminal paper dealing with the ac fields of a submerged source for the three-layer case is that of Weaver (1967). An excellent compendium of simplified formulas for the two-layer case is given in Kraichman's book (1976) and further formulas for this model have

Correspondence to: D. Llanwyn Jones (email) david.jones@bay.cc.kcl.ac.uk been published in a series of papers by Bannister (see e.g. Bannister, 1984). Developments in relation to the ocean/lithosphere environment have been presented, for example, by Chave and Cox (1982) and Fraser-Smith et al. (1988). Often in geophysics, point current sources and sinks are used to produce subsurface fields for remote sensing, and the configuration used is such that the source acts essentially as a dipole (Wait 1982, 1993). An important feature of the ac formulation is the presence of 'lateral waves' which aid the propagation of the fields. These waves are not present in the dc case. A large number of relevant mathematical and practical results for ac and dc are presented by Wait (1982), but the dc formulas in this paper (for the three-layer case) have not been published previously. Here we concentrate upon these dc (or static) solutions which apply for vanishing small values of $r / \lambda$.

The mathematical background to our formulation is that developed by Sommerfeld (1967) and, particularly, Wait (1987). In Burke and Jones (1992, 1993, 1994), we considered an ac radiation source located in seawater the central region of a three-layered horizontally stratified medium. We assumed a time-harmonic source dipole moment of angular frequency $\omega$, and presented integral formulas of the Sommerfeld type for the twocomponent Hertz vector $\left(\Pi_{x}, 0, \Pi_{z}\right)$ which is required for the solution in the case of horizontal electric dipole (HED) radiation source and the single-component Hertz vector $\left(0,0, \Pi_{z}\right)$ needed for the vertical electric dipole (VED). We also gave explicit formulas from which the Cartesian components of the $\boldsymbol{E}$ and $\boldsymbol{B}$ fields can be computed by quadrature. The field expressions are the sum of two parts - an analytic primary contribution which is the field produced in a medium of infinite extent, and an integral secondary contribution arising from the presence of the interfaces. These previous formulas are valid at any wave frequency, including, particularly, the dc case, $\omega=0$.

For the dc case, we show that the integral formulas can be evaluated analytically using Laplace transforms. This process leads to an infinite series expansion suitable 
for computation. Each term of the infinite series corresponds to a particular image of the source in the upper or the lower interface. Needless to say, the application of the infinite series produces a much faster algorithm for computing the field components at dc than any involving numerical integration.

It is customary to determine de solutions by deriving formulas for a scalar electric potential and a vector magnetic potential. In this paper, however, we derive dc analytic formulas from our pre-existing ac equations derived from a Hertz vector. In part, this work was undertaken to validate our ac field derivations and computer code (Burke and Jones, 1992, 1993). The results for the various dc field components are given in a compact tabular form for reference and computational purposes. We also give specimen numerical data.

\section{The physical model}

We first consider an HED of current moment $\operatorname{Id} \ell$ ( Am) placed in the central (conducting) layer of a three-layer, horizontally stratified medium. The upper region is taken to be free space and the lower two regions are conductors with conductivities $\sigma_{2}$ and $\sigma_{3}(\mathrm{~S} / \mathrm{m})$, respectively. The three regions could represent a geophysical air-overburden-basement or an oceanic air-sea- seabed problem. Cartesian co-ordinates are used with $z$ directed downwards, $z=0$ being the upper interface. The magnetic permeability is assumed to be that of free space everywhere, so the dc fields of a magnetic dipole source are unaffected by the interfaces and are the same as those of a magnetic dipole located in free space. The three layers are of infinite extent in the $x$ and $y$ directions. The geometry is illustrated in Fig. 1.

The HED source is $x$-directed and located at $(0,0, h)$. The central region is of thickness $d$; the upper and lower regions are assumed to be of infinite thickness. Fields are to be computed at the point $(x, y, z)$ with $-\infty \leq z \leq d$, i.e. anywhere in layers 1 or 2 . If it is assumed that $\sigma_{2}=\sigma_{3}$ (or, alternatively, that $d \rightarrow \infty$ ), only the upper interface affects the solution. This is termed the 'twolayer' or 'thick-layer' solution in distinction to the 'thinlayer' solution obtained for finite $d$ for which both interfaces (upper and lower) materially affect the field components.

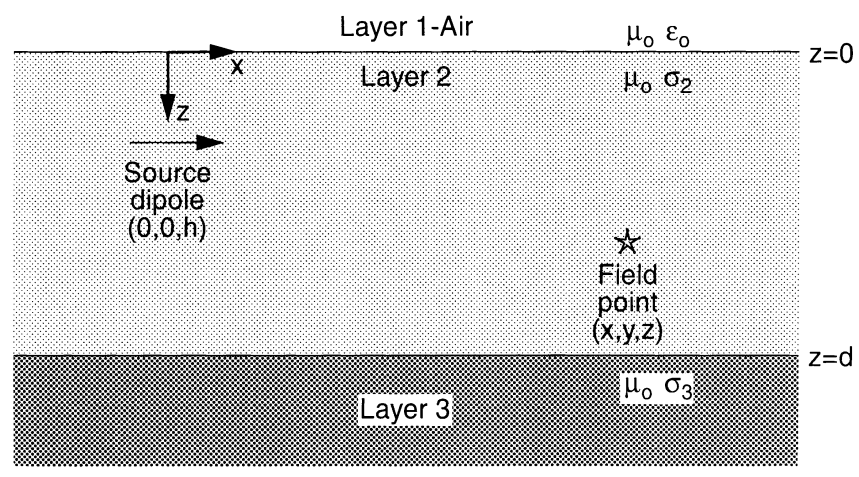

Fig. 1. The three-layer geometry

\section{The field equations for the HED}

\subsection{Derivation of the HED dc field equations}

In Burke and Jones, 1992, $§ 3$ (henceforth Ref. 1), general equations were presented for the two components of the Hertz vector in layer $i, \Pi_{x i}$ and $\Pi_{z i}$, produced by an HED source dipole located in layer $i=2$. Using the notation of this previous paper, we now consider the limit $\omega \rightarrow 0$ to deduce the appropriate formulas for the dc case. When the formulas for the components of the Hertz vector have been established, the static $\boldsymbol{E}$ and $\boldsymbol{H}$ field vectors can be computed in any layer from the standard expression (e.g. Stratton, 1941),

$\boldsymbol{E}=k^{2} \boldsymbol{\Pi}+\operatorname{grad} \operatorname{div} \boldsymbol{\Pi}$,

$\boldsymbol{H}=\sigma^{*} \operatorname{curl} \boldsymbol{\Pi}$,

where $k^{2}=-i \omega \mu_{0} \sigma^{*}$ and $\sigma^{*}=(\sigma+i \omega \epsilon)$ is the complex conductivity of the layer in which the field is to be computed. At dc, in layer $2, \sigma^{*}=\sigma_{2}$ and is real, but in layer $1, \sigma^{*}=i \omega \epsilon_{0}$, and some care has to be taken in evaluating the limit of the right-hand side of Eq. (1) as $\omega \rightarrow 0$.

\subsubsection{HED fields in layer 2}

At dc, the Sommerfeld parameter $u=\left(\lambda^{2}-k^{2}\right)^{\frac{1}{2}}$ which appears in the ac formulation (Ref. 1) may be replaced by $\lambda$. Thus, for the dc case, the components of the Hertz vector in layer 2 are written in the form,

$$
\begin{aligned}
& \Pi_{x 2}=M \int_{0}^{\infty}\left(e^{-\lambda|z-h|}+B e^{-\lambda z}+C e^{\lambda z}\right) J_{0}(\lambda \rho) d \lambda, \\
& \Pi_{z 2}=M \frac{\partial}{\partial x} \int_{0}^{\infty}\left(T e^{-\lambda z}+S e^{\lambda z}\right) J_{0}(\lambda \rho) d \lambda,
\end{aligned}
$$

where $M=I d \ell /\left(4 \pi \sigma_{2}\right), \rho^{2}=x^{2}+y^{2}$ and $J_{0}$ is the Bessel function of the first kind of order zero.

Explicit expressions for the four coefficients $B, C, S$ and $T$ were given in Ref. 1. These expressions involve $d$, $h$ and $z$, together with the layer conductivities which enter the equations in terms of the interfacial reflection coefficients for horizontal $\left(R_{i j}\right)$ and vertical polarisation $\left(R_{i j}^{\|}\right)$for fields incident from layer $i$ onto the interface with layer $j$. It is immediately seen from the results of Ref. 1 that as $\omega \rightarrow 0$,

$R_{21}=R_{23}=0$,

and

$R_{21}^{\|}=-1, R_{23}^{\|}=\left(\sigma_{3}-\sigma_{2}\right) /\left(\sigma_{3}+\sigma_{2}\right)$.

It follows from the first two of these that the coefficients $B$ and $C$ in the expression for $\Pi_{x 2}$ are both zero, so that $\Pi_{x 2}$ is unaffected by the presence of the interfaces. It then follows from Sommerfeld's ac formula [Sommerfeld, 1967, p242, Eq. (14)] that, at dc, $\Pi_{x 2}$ is expressible in closed form: 
$\Pi_{x 2}=M \int_{0}^{\infty} e^{-\lambda|z-h|} J_{0}(\lambda \rho) d \lambda=M\left[\rho^{2}+(h-z)^{2}\right]^{-\frac{1}{2}}$.

It is noted that this integral can be also be represented as a known Laplace transform; see, for example, Abramowitz and Stegun (1968, Transform \#29.3.55, p. 1024), which gives a direct way of obtaining the result of Eq. (6) for the dc case. Equation (6) gives the primary field of the dipole, i.e. the field of a dipole located in a medium of infinite extent.

When the differential in Eq. (4) is evaluated, the expression for $\Pi_{z 2}$ becomes

$\Pi_{z 2}=-M \frac{x}{\rho} \int_{0}^{\infty} \lambda\left(S e^{\lambda z}+T e^{-\lambda z}\right) J_{1}(\lambda \rho) d \lambda$,

$J_{1}$ being the Bessel function of the first kind of order one. At dc, the coefficients $S$ and $T$ are (Ref. 1)

$S=-\left(R_{23}^{\|} / D^{\|} \lambda\right)\left(e^{-\lambda(2 d+h)}+e^{-\lambda(2 d-h)}\right)$

and

$T=-\left(1 / D^{\|} \lambda\right)\left(e^{-\lambda h}-R_{23}^{\|} e^{-\lambda(2 d-h)}\right)$,

where $D^{\|}=1+R_{23}^{\|} e^{-2 \lambda d}$.

We now expand the factor $1 / D^{\|}$as a geometric progression

$1 / D^{\mid}=\sum_{n=0}^{\infty}\left(-R_{23}^{\|}\right)^{n} e^{-2 n d \lambda}$.

It then follows that $\Pi_{z 2}$ can be expressed as the sum of four terms:

$\Pi_{z 2}=\sum_{i=1}^{4} \Pi_{z 2}^{(i)}=\Pi_{z 2}^{(1)}+\Pi_{z 2}^{(2)}+\Pi_{z 2}^{(3)}+\Pi_{z 2}^{(4)}$,

with

$\Pi_{z 2}^{(1)}=+M \frac{x}{\rho} \int_{0}^{\infty} e^{-\lambda(h+z)} \sum_{n=0}^{\infty}\left(-R_{23}^{\|}\right)^{n} e^{-2 n d \lambda} J_{1}(\lambda \rho) d \lambda$,

$\Pi_{z 2}^{(2)}=-M \frac{x}{\rho} \int_{0}^{\infty} R_{23}^{\|} e^{-\lambda(2 d-h+z)} \sum_{n=0}^{\infty}\left(-R_{23}^{\|}\right)^{n} e^{-2 n d \lambda} J_{1}(\lambda \rho) d \lambda$,

$\Pi_{z 2}^{(3)}=+M \frac{x}{\rho} \int_{0}^{\infty} R_{23}^{\|} e^{-\lambda(2 d+h-z)} \sum_{n=0}^{\infty}\left(-R_{23}^{\|}\right)^{n} e^{-2 n d \lambda} J_{1}(\lambda \rho) d \lambda$,

$\Pi_{z 2}^{(4)}=+M \frac{x}{\rho} \int_{0}^{\infty} R_{23}^{\|} e^{-\lambda(2 d-h-z)} \sum_{n=0}^{\infty}\left(-R_{23}^{\|}\right)^{n} e^{-2 n d \lambda} J_{1}(\lambda \rho) d \lambda$.

Consider the term $\Pi_{z 2}^{(1)}$. This may be written as

$\Pi_{z 2}^{(1)}=M \frac{x}{\rho} \int_{0}^{\infty} \sum_{n=0}^{\infty}\left(-R_{23}^{\|}\right)^{n} \exp \left(-\lambda r_{n 1}\right) J_{1}(\lambda \rho) d \lambda$,

where $r_{n 1}=2 n d+h+z$.
As was the case for Eq. (6), the integral in Eq. (11) can be evaluated using a tabulated result for the Laplace transform (Abramowitz and Stegun, 1968, Transform \#29.3.56, p. 1024) viz:

$\int_{0}^{\infty} a e^{-s t} J_{1}(a t) d t=\left(\sqrt{s^{2}+a^{2}}-s\right)\left(\sqrt{s^{2}+a^{2}}\right)^{-1}$.

For small values of $a / s$, greater numerical accuracy is obtained by recasting the right-hand side of this formula using the identity:

$$
\begin{aligned}
& \left(\sqrt{s^{2}+a^{2}}-s\right)\left(\sqrt{s^{2}+a^{2}}\right)^{-1} \\
& \equiv a^{2}\left(s^{2}+a^{2}+s \sqrt{s^{2}+a^{2}}\right)^{-1} .
\end{aligned}
$$

Putting $a=\rho, t=\lambda$ and $s=r_{n 1}$, it follows that

$$
\Pi_{z 2}^{(1)}=+M x \sum_{n=0}^{\infty}\left(-R_{23}^{\|}\right)^{n} /\left(r_{n 1}^{2}+\rho^{2}+r_{n 1} \sqrt{r_{n 1}^{2}+\rho^{2}}\right) \text {. }
$$

It is noted that for a thick central layer, with $h$ and $z$ finite so that $d \gg h+\rho+z$, only the $n=0$ term of this series expansion survives. This term is that produced by the image of the source dipole in the upper interface and is a secondary field component. Further secondary components are produced by the higher values of $n$.

Applying the same technique to the remaining terms in Eq. (10), we obtain

$\Pi_{z 2}^{(2)}=+M x \sum_{n=1}^{\infty}\left(-R_{23}^{\|}\right)^{n} /\left(r_{n 2}^{2}+\rho^{2}+r_{n 2} \sqrt{r_{n 2}^{2}+\rho^{2}}\right)$,

$\Pi_{z 2}^{(3)}=-M x \sum_{n=1}^{\infty}\left(-R_{23}^{\|}\right)^{n} /\left(r_{n 3}^{2}+\rho^{2}+r_{n 3} \sqrt{r_{n 3}^{2}+\rho^{2}}\right)$,

$\Pi_{z 2}^{(4)}=-M x \sum_{n=1}^{\infty}\left(-R_{23}^{\|}\right)^{n} /\left(r_{n 4}^{2}+\rho^{2}+r_{n 4} \sqrt{r_{n 4}^{2}+\rho^{2}}\right)$,

with $r_{n 2}=2 n d-h+z, r_{n 3}=2 n d+h-z$ and $r_{n 4}=2 n d$ $-h-z$.

Note that in Eq.(12b-d) the summations do not include the term $n=0$. These three terms thus make no contribution to the solution when $d$ is very large, provided that $h$ and $z$ are finite.

The solution presented is, in fact, a geometricaloptics (or images) formula which, as is well known, is valid when the reflection coefficient is independent of the 'angle of incidence'. In the given formulation this angle of incidence is embodied within the Sommerfeld 'dummy' variable $\lambda$. The images associated with a positive sign preceding $z$ in the formulas for $r_{n i}$ are in layer 1 and those with a negative sign are in layer 3 .

Equations (6) and (12) are the complete solution for the Hertz vector $\Pi$ in layer 2 . The field components in this layer can now be derived using Eqs. (1) and (2). The results of this tedious process are presented in Sect. 4.1. 


\subsubsection{HED dc fields in layer 1}

Again, referring to the ac formulas presented in Ref. 1, it is easily seen that, at dc, the Hertz vector components $\Pi_{x 1}$ and $\Pi_{z 1}$ in the air-space (layer 1) may be represented in the form

$$
\begin{aligned}
\Pi_{x 1} & =M \int_{0}^{\infty} A e^{\lambda z} J_{0}(\lambda \rho) d \lambda, \\
\Pi_{z 1} & =M \frac{\partial}{\partial x} \int_{0}^{\infty} R e^{\lambda z} J_{0}(\lambda \rho) d \lambda, \\
& =-M \frac{x}{\rho} \int_{0}^{\infty} \lambda R e^{\lambda z} J_{1}(\lambda \rho) d \lambda .
\end{aligned}
$$

Application of the boundary conditions across the interfaces $z=0$ and $z=d$ (as described in Ref. 1) produces the following results for the two coefficients $A$ and $R$ as $\omega \rightarrow 0$ :

$A=\left(-i \sigma_{2} / \omega \varepsilon_{0}\right) e^{-\lambda h}$

$R=\left(-i \sigma_{2} / \omega \varepsilon_{0}\right)(S+T)=(1 / \lambda)\left(i \sigma_{2} / \omega \varepsilon_{0}\right) e^{-\lambda h}$.

Hence, as $\omega \rightarrow 0$, both $A$ and $R$, and thus $\Pi_{x 1}$ and $\Pi_{z 1} \rightarrow \infty$. However, Eqs. (1) and (2) must give finite values for the dc fields $\boldsymbol{E}$ and $\boldsymbol{H}$. In Eq. (1) $k^{2} \boldsymbol{\Pi} \rightarrow 0$ and in Eq. (2) $i \omega \varepsilon_{0} \nabla \times \boldsymbol{\Pi}$ is clearly finite. We will now show that in Eq. (1), $\nabla \nabla \cdot \Pi$ is also finite.

3.1.2.1 Calculation of $\nabla \cdot \boldsymbol{\Pi}$ and hence $\boldsymbol{E}$. From Eqs. (13) and (14) and the elementary formula

$\nabla \cdot \boldsymbol{\Pi}=\partial \Pi_{x} / \partial x+\partial \Pi_{z} / \partial z$

it is found that

$\nabla \cdot \boldsymbol{\Pi}=-M \frac{x}{\rho} \int_{0}^{\infty} \lambda(A+\lambda R) e^{\lambda z} J_{1}(\lambda \rho) d \lambda$.

In Eq. (16) the factor $A+\lambda R$ is indeterminate as $\omega \rightarrow 0$ if Eq. (15) is used. To resolve this indeterminacy we return to the boundary condition that both $E_{x}$ and $E_{y}$ and hence $\nabla \cdot \Pi$ is continuous across the interface $z=0$. At dc this requires that

$$
\begin{aligned}
A+\lambda R & =e^{-\lambda h}+\lambda(S-T) \\
& =\left(2 / D^{\|}\right)\left(e^{-\lambda h}-R_{23}^{\|} e^{-\lambda(2 d-h)}\right),
\end{aligned}
$$

and thus

$$
\begin{aligned}
\nabla \cdot \boldsymbol{\Pi}=-2 M \frac{x}{\rho} \int_{0}^{\infty} & \left(\lambda / D^{\|}\right)\left(e^{-\lambda h}-R_{23}^{\|} e^{-\lambda(2 d-h)}\right) \\
& \times e^{\lambda z} J_{1}(\lambda \rho) d \lambda .
\end{aligned}
$$

In this formula the integrand may be cast into the same form as that previously expanded [Eq. (10)] by noting that it is equivalent to

$$
\begin{aligned}
\nabla \cdot \boldsymbol{\Pi}=-2 M \frac{x}{\rho} \frac{\partial}{\partial z} \int_{0}^{\infty} & \left(1 / D^{\|}\right)\left(e^{-\lambda(h-z)}-R_{23}^{\|} e^{-\lambda(2 d-h-z)}\right) \\
& \times J_{1}(\lambda \rho) d \lambda .
\end{aligned}
$$

Proceeding as in Sect. 3.1.1, we then find the following series expansion formula for Eq.(18).

$$
\begin{aligned}
\nabla \cdot \boldsymbol{\Pi}= & -2 M x \frac{\partial}{\partial z}\left[\sum_{n=0}^{\infty}\left(-R_{23}^{\|}\right)^{n} /\left(r_{n 3}^{2}+\rho^{2}+r_{n 3} \sqrt{r_{n 3}^{2}+\rho^{2}}\right)\right. \\
& \left.+\sum_{n=1}^{\infty}\left(-R_{23}^{\|}\right)^{n} /\left(r_{n 4}^{2}+\rho^{2}+r_{n 4} \sqrt{r_{n 4}^{2}+\rho^{2}}\right)\right] .
\end{aligned}
$$

By evaluating the gradient of Eq. (19) in accordance with Eq. (1), we obtain the series expansion formulas for the electric field components in layer 1 . These are given in Sect.4.2. Note that the $n=0$ term in Eq. (19) represents the sum of the primary (or direct) field plus the 'thick-layer' field corresponding to $d \rightarrow \infty$, these being equal for a point in layer 1 . Also only the images in layer 3 contribute to the field in layer $1\left[r_{n 1}\right.$ and $r_{n 2}$ are absent in Eq. (19)].

3.1.2.2 Calculation of $\boldsymbol{H}$ (or $\boldsymbol{B})$. $\boldsymbol{H}$ is given by Eq. (2) with $\sigma^{*}=i \omega \varepsilon_{0}$ for layer 1. From Eq. (15), $i \omega \varepsilon_{0} A=\sigma_{2} e^{-\lambda h}$ and $i \omega \varepsilon_{0} R=-\sigma_{2}(1 / \lambda) e^{-\lambda h}$. It then follows that [as for Eq. (6)],

$$
\begin{aligned}
i \omega \varepsilon_{0} \Pi_{x 1} & =M \sigma_{2} \int_{0}^{\infty} e^{-\lambda(h-z)} J_{0}(\lambda \rho) d \lambda \\
& =M \sigma_{2}\left[\rho^{2}+(h-z)^{2}\right]^{-\frac{1}{2}},
\end{aligned}
$$

and

$$
\begin{aligned}
i \omega \varepsilon_{0} \Pi_{z 1}= & M \sigma_{2} \frac{x}{\rho} \int_{0}^{\infty} e^{-\lambda(h-z)} J_{1}(\lambda \rho) d \lambda=M \sigma_{2} x \\
& \times\left(\rho^{2}+(h-z)^{2}+(h-z)\left[\rho^{2}+(h-z)^{2}\right]^{\frac{1}{2}}\right)^{-1} .
\end{aligned}
$$

The magnetic field components in layer 1 may now be computed as $\boldsymbol{H}=\nabla \times\left(i \omega \varepsilon_{0} \boldsymbol{\Pi}\right)$, the $x$ and $z$ components of the vector $\Pi$ being given by the closed form expressions, (20) and (21). Interestingly, these dc magnetic field components are independent of $d, \sigma_{2}$ and $\sigma_{3}$ (note that $M \sigma_{2}=I d \ell / 4 \pi$ ). The field formulas derived from Eqs. (20) and (21) are given in Sect. 4.2.

\section{Recipes for computing the HED dc field components}

\subsection{HED DC Fields in Layer 2}

The field equations for computing $\boldsymbol{E}$ and $\boldsymbol{B}=\mu_{0} \boldsymbol{H}$, derived from the formulas of Sect. 3 are given in Tables 1 and 2. The field components are computed using the tables as follows.

a) First compute the primary (direct) field produced by the source dipole using the equations in Table 1 , [derived from Eq. (6)]. These are the fields produced by the source if located in a medium of conductivity $\sigma_{2}$ which is of infinite thickness. These results are well known and can be put in the form presented in the table using formulas presented in many standard texts (e.g. Stratton, 1941, p. 436).

b) Secondly calculate the secondary field components for the case when the middle layer is of infinite thickness 
Table 1. Layer-2 primary fields of the HED

\begin{tabular}{l}
$E_{x}=E_{0}\left(3 x^{2} / D^{5}-1 / D^{3}\right)$ \\
$E_{y}=E_{0} 3 x y / D^{5}$ \\
$E_{z}=E_{0} 3 x(z-h) / D^{5}$ \\
$B_{x}=0$ \\
$B_{y}=-B_{0}(z-h) / D^{3}$ \\
$B_{z}=B_{0} y / D^{3}$ \\
\hline$E_{0}=10^{6} I d \ell /\left(4 \pi \sigma_{2}\right) \quad($ giving $\boldsymbol{E}$ in $\mu \mathrm{V} / \mathrm{m})$ \\
$B_{0}=100 \mathrm{Id} \ell$ \\
$D^{2}=\rho^{2}+(z-h)^{2}, \rho^{2}=x^{2}+y^{2}$
\end{tabular}

(the 'thick-layer' case). This is done by using the formulas of Table 2 with $i=1$ and $n=0$. These formulas are obtained from the $n=0$ term of Eq. (12a). The only value of $r_{n i}$ required is thus

$r_{01}=h+z$.

Add these field values to those obtained in stage (a). The result is that for the fields produced by a source at finite depth within an infinitely thick central layer, and the fields are thus affected by the upper interface only. The source of this term is the image dipole located in layer 1 at a distance $h$ above the upper interface.

c) To find the field for the general thin-layer or threelayer case the results of Table 2 are used in the following manner. If $F$ represents any one of the field formulas given in the second column of Table 2 compute the double summation:

$\sum_{n=1}^{\infty}\left[\sum_{i=1}^{4} F_{n i}\right]$.

Repeat this calculation for the five finite components in Table 2. The convergence of the summation depends on the value of $R$ (which is the reflection coefficient of the

Table 2. Layer-2 secondary field terms for the HED

\begin{tabular}{|c|c|c|}
\hline$E_{x}$ & $-E_{0}(-R)^{n} \frac{r_{n i}^{2}+\rho^{2}-3 x^{2}}{\left(r_{n i}^{2}+\rho^{2}\right)^{5 / 2}}$ & \\
\hline$E_{y}$ & $+E_{0}(-R)^{n} \frac{3 x y}{\left(r_{n i}^{2}+\rho^{2}\right)^{5 / 2}}$ & \\
\hline$E_{z}$ & $+S_{i} E_{0}(-R)^{n} \frac{3 x r_{n i}}{\left(r_{n i}^{2}+\rho^{2}\right)^{5 / 2}}$ & $\begin{array}{l}S_{i}=+1, i=1,2 \\
S_{i}=-1, i=3,4\end{array}$ \\
\hline$B_{x}$ & $+S_{i} B_{0}(-R)^{n} \frac{x y}{\rho^{4}}\left[\frac{r_{n i}\left(2 r_{n i}^{2}+3 \rho^{2}\right)}{\left(r_{n i}^{2}+\rho^{2}\right)^{3 / 2}}-2\right]$ & $\begin{array}{l}S_{i}=+1, i=1,2 \\
S_{i}=-1, i=3,4\end{array}$ \\
\hline$B_{y}$ & $-S_{i} B_{0}(-R)^{n}\left\{\frac{x^{2}}{\rho^{4}}\left[\frac{r_{n i}\left(2 r_{n i}^{2}+3 \rho^{2}\right)}{\left(r_{n i}^{2}+\rho^{2}\right)^{3 / 2}}-2\right]\right.$ & $\begin{array}{l}S_{i}=+1, i=1,2 \\
S_{i}=-1, i=3,4\end{array}$ \\
\hline & 1 & \\
\hline & $\left.+\overline{r_{n i}^{2}+\rho^{2}+r_{n i}\left(r_{n i}^{2}+\rho^{2}\right)^{1 / 2}}\right\}$ & \\
\hline$B_{z}$ & 0 & \\
\hline
\end{tabular}

$E_{0}, B_{0}$ and $\rho$ are as defined in Table 1

$R=\left(\sigma_{3}-\sigma_{2}\right) /\left(\sigma_{3}+\sigma_{2}\right)$

$r_{n 1}=2 n d+h+z, r_{n 2}=2 n d-h+z$,

$r_{n 3}=2 n d+h-z, r_{n 4}=2 n d-h-z, n=1,2,3 \ldots$

Note: As $x \rightarrow 0$ and $y \rightarrow 0$ so $\rho \rightarrow 0 ; B_{x} \rightarrow 0$ and only the second term in the \{\} for $B_{y}$ is finite. lower interface, $R_{23}^{\|}$) and on the spatial parameters, particularly the layer thickness $d$ ( $R$ small and/or $d$ large gives rapid convergence). The double summation computes the fields given by the $n>0$ terms of Eq. (12) which are the fields of the multiple images of the source in the upper $(i=1,2)$ and lower $(i=3,4)$ layers.

The final result is obtained by adding the field produced in step (c) to that produced at step (b). If the $i$ summation is first computed for $n=1$ and the result added to that of step (b), the convergence can be tested following each successive $n$-summation enabling a simple algorithm to be devised for terminating the summation with a machine of any given precision.

d) Note: if the middle layer is infinitely thick but the source dipole is near the base so that $h^{\prime}=d-h$ $z^{\prime}=d-z$ are finite, the fields can be computed as the primary field [as in (a)] plus a secondary term which is the $i=4, n=1$ term in Table 2. This follows because $r_{14}=2 d-h-z=h^{\prime}+z^{\prime}$ is the only finite $r_{n i}$ in this situation.

\subsection{HED dc fields in the upper region (layer 1)}

The fields in the upper region (produced by a source in layer 2) are computed as follows. Note that as $z<0$, a negative value of $z$ is to be used for all calculations in this region.

a) Compute the primary fields from the formulas of Table 1.

b) For the electric field components, the secondary fields for the two-layer (or 'thick-layer') case are the same as the primary fields. Thus, for the electric components, multiply the results obtained in (a) by two to get the primary plus 'thick layer' contributions.

For the magnetic field components, the secondary fields for the two-layer case can be computed using the B-formulas of Table 2 with $S_{i}=+1$ and replacing $r_{n i}$ by $h-z$ (remember that a negative value of $z$ is to be used so $h-z$ is always a positive quantity). The calculated values are then added to those produced by step (a).

c) The calculation is now complete for the magnetic fields because there are no 'thin-layer' contributions.

In the case of the electric fields the secondary 'thinlayer' contribution to be added to that produced in step (b) is calculated by using the formulas of Table 3 and computing the double summation

$\sum_{n=1}^{\infty}\left[\sum_{i=3}^{4} F_{n i}\right]$.

Table 3. Layer-1 secondary field terms for the HED

\begin{tabular}{lll}
\hline$E_{x}$ & $-2 S_{i} E_{0}(-R)^{n} \frac{r_{n i}^{2}+\rho^{2}-3 x^{2}}{\left(r_{n i}^{2}+\rho^{2}\right)^{5 / 2}}$ & $S_{3}=+1$ \\
$E_{y}$ & $+2 S_{i} E_{0}(-R)^{n} \frac{3 x y}{\left(r_{n i}^{2}+\rho^{2}\right)^{5 / 2}}$ & $S_{4}=-1$ \\
& & $S_{3}=+1$ \\
$E_{z}$ & $-2 S_{i} E_{0}(-R)^{n} \frac{3 x r_{n i}}{\left(r_{n i}^{2}+\rho^{2}\right)^{5 / 2}}$ & $S_{4}=-1$ \\
& & $S_{3}=+1$ \\
& & $S_{4}=-1$
\end{tabular}


(Note that the second summation is only taken over $i=3$ and 4 in this situation. Except for the sign and a factor of two, the formulas in Table 3 are the same as those in Table 2.)

\section{The vertical electric dipole}

The vertical electric dipole ( VED) is taken to be $z$ directed (i.e. directed downwards) and located at the point $(0,0, h)$. Otherwise the situation is as described in Sect. 2 .

Because, in this case, there is complete azimuthal symmetry, the field formulas are less involved than is the case for the HED. The boundary conditions can be satisfied with a single-component Hertz vector $\Pi_{z i}(i=1,2,3)$ in each of the three layers, which is written in the same form as that for $\Pi_{x i}$ for the HED. Initially it is advantageous to use cylindrical polar coordinates $(\rho, \varphi, z)$. The cylindrical polar components evaluated using Eqs. (1) and (2) are, explicitly:

$E_{\rho}=\partial^{2} \Pi_{z} / \partial \rho \partial z, E_{z}=\left(k^{2}+\frac{\partial^{2}}{\partial z^{2}}\right) \Pi_{z}, H_{\varphi}=-\sigma^{*} \partial \Pi_{z} / \partial \rho$

all other field components being zero. The boundary conditions for the VED thus reduce to the continuity of $k^{2} \Pi_{z}$ and $\partial \Pi_{z} / \partial z$ across each of the two interfaces. The solution for the VED at dc then proceeds analogously to that for the HED and we will not detail the algebraic processes involved here.

\subsection{VED dc fields in layer 2}

It is found that, at dc, the Hertz vector in layer 2 is expressible in the form of Eq. (10a) with

$$
\begin{aligned}
& \Pi_{z 2}^{(1)}=-M \sum_{n=0}^{\infty}\left(-R_{23}^{\|}\right)^{n} / \sqrt{r_{n 1}^{2}+\rho^{2}}, \\
& \Pi_{z 2}^{(2)}=+M \sum_{n=1}^{\infty}\left(-R_{23}^{\|}\right)^{n} / \sqrt{r_{n 2}^{2}+\rho^{2}}, \\
& \Pi_{z 2}^{(3)}=+M \sum_{n=1}^{\infty}\left(-R_{23}^{\|}\right)^{n} / \sqrt{r_{n 3}^{2}+\rho^{2}}, \\
& \Pi_{z 2}^{(4)}=-M \sum_{n=1}^{\infty}\left(-R_{23}^{\|}\right)^{n} / \sqrt{r_{n 4}^{2}+\rho^{2}},
\end{aligned}
$$

from which the field components in layer 2 can be computed using Eq. (22). The results are presented in Sect. 6.1.

\subsection{VED dc fields in layer 1}

In layer 1 , it is found that $\Pi_{z 1}$ may be written as [compare Eq. (19) for the HED, noting that $\nabla \cdot \boldsymbol{\Pi}_{1}=$ $\left.\partial \Pi_{z 1} / \partial z\right]$ :

$$
\begin{aligned}
\Pi_{z 1}=2 M[ & \sum_{n=0}^{\infty}\left(-R_{23}^{\|}\right)^{n} / \sqrt{r_{n 3}^{2}+\rho^{2}} \\
& \left.-\sum_{n=1}^{\infty}\left(-R_{23}^{\|}\right)^{n} / \sqrt{r_{n 4}^{2}+\rho^{2}}\right] .
\end{aligned}
$$

In Eqs. (23) and (24) the $r_{n i}$ (with $i=3,4$ ) are defined following Eq. (12). In Eq. (22), for layer $1, \sigma^{*} \equiv 0$ at dc (and $\Pi_{z 1}$ is finite). It follows that $H_{\varphi}$ is zero in this layer. This is to be expected from Amperes law, because of the symmetry and the fact that there are no currents in layer 1 . The electric field components, as computed from Eqs. (22) and (24) are presented in Sect. 6.2.

\section{Recipes for computing the VED dc field components}

\subsection{VED fields in layer 2}

The formulas for the VED fields in layer 2 are listed in Tables 4 and 5. The results in Table 4 are those of Table 1 modified for a $z$-directed dipole. Computation proceeds exactly as described in Sect. 4.1 except that Tables 4 and 5 are used in place of Tables 1 and 2, respectively. The cylindrical polar components $\left(E_{\rho}, B_{\varphi}\right.$, $E_{z}$ ) should first be calculated to the precision required. Finally, if needed, the Cartesian components may be computed from these using the relations presented in Table 4.

Table 4. Layer-2 primary fields of the VED

$$
\begin{aligned}
& E_{\rho}=E_{0} 3 \rho(z-h) / D^{5} \\
& E_{z}=E_{0}\left[3(z-h)^{2}-D^{2}\right] / D^{5} \\
& B_{\varphi}=B_{0} \rho / D^{3} \\
& E_{x}=x E_{\rho} / \rho: E_{y}=y E_{\rho} / \rho \\
& B_{x}=-y B_{\varphi} / \rho: B_{y}=x B_{\varphi} / \rho: B_{z}=0 \\
& \left.E_{0}=10^{6} I d \ell /\left(4 \pi \sigma_{2}\right) \quad \text { (giving } \boldsymbol{E} \text { in } \mu \mathrm{V} / \mathrm{m}\right) \\
& B_{0}=100 I d \ell \\
& D^{2}=\rho^{2}+(z-h)^{2}, \rho^{2}=x^{2}+y^{2}
\end{aligned}
$$

Table 5. Layer-2 secondary field terms for the VED

\begin{tabular}{lll}
\hline$E_{\rho}$ & $-S_{i} E_{0}(-R)^{n} \frac{3 \rho r_{n i}}{\left(r_{n i}^{2}+\rho^{2}\right)^{5 / 2}}$ & $S_{i}=+1, i=1,3$ \\
& & $S_{i}=-1, i=2,4$ \\
$E_{z}$ & $-S_{i} E_{0}(-R)^{n} \frac{2 r_{n i}^{2}-\rho^{2}}{\left(r_{n i}^{2}+\rho^{2}\right)^{5 / 2}}$ & $S_{i}=+1, i=1,4$ \\
& & $S_{i}=-1, i=2,3$ \\
$B_{\varphi}$ & $-S_{i} B_{0}(-R)^{n} \frac{\rho}{\left(r_{n i}^{2}+\rho^{2}\right)^{3 / 2}}$ & $S_{i}=+1, i=1,4$ \\
& & $S_{i}=-1, i=2,3$ \\
\hline
\end{tabular}

For $E_{x}, E_{y}, B_{x}$ and $B_{y}$ use the formulas in Table 4;

$E_{0}, B_{0}$ and $\rho$ are as defined in Table 4;

$R=\left(\sigma_{3}-\sigma_{2}\right) /\left(\sigma_{3}+\sigma_{2}\right)$.

$r_{n 1}=2 n d+h+z, r_{n 2}=2 n d-h+z$,

$r_{n 3}=2 n d+h-z, r_{n 4}=2 n d-h-z, n=1,2,3 \ldots$ 


\subsection{VED fields in layer 1}

In layer 1 there are no magnetic field components for the VED (i.e. $\boldsymbol{B}=0$ ) so only the electric fields have to be computed. Computations of the electric field components are made following the procedure described in Sect. 4.2 using the $E$-field formulas of Tables 4 and 5 as follows.

To compute the sum of the primary plus thick-layer contribution use Table 4, multiplying each of the two electric field components by two. Then add on the result of the summation over $n$ for $i=3$ and 4 (as in Sect. 4.2c) using the $E$-formulas of Table 5, but again multiplying each field component in Table 5 by two.

\section{Specimen calculations and validation}

\subsection{Specimen Calculations}

Specimen calculations for the HED and the VED gave the results presented in Table 6. A single precision algorithm was used and the computation terminated automatically (e.g. at $n=31$ for the layer 2 fields for the HED when the largest field component increment was, at most, $0.5 \times 10^{-5}$ the existing value of that component)

\subsection{Validation}

We have tested the dc formulas presented in this paper against our ac model (Burke and Jones, 1993, 1994), which had previously been programmed. This process checks the analytic derivations of the field components from the Hertz vector for the two cases and the resulting computer code. Clearly, the ac model should give the

Table 6. Specimen calculations

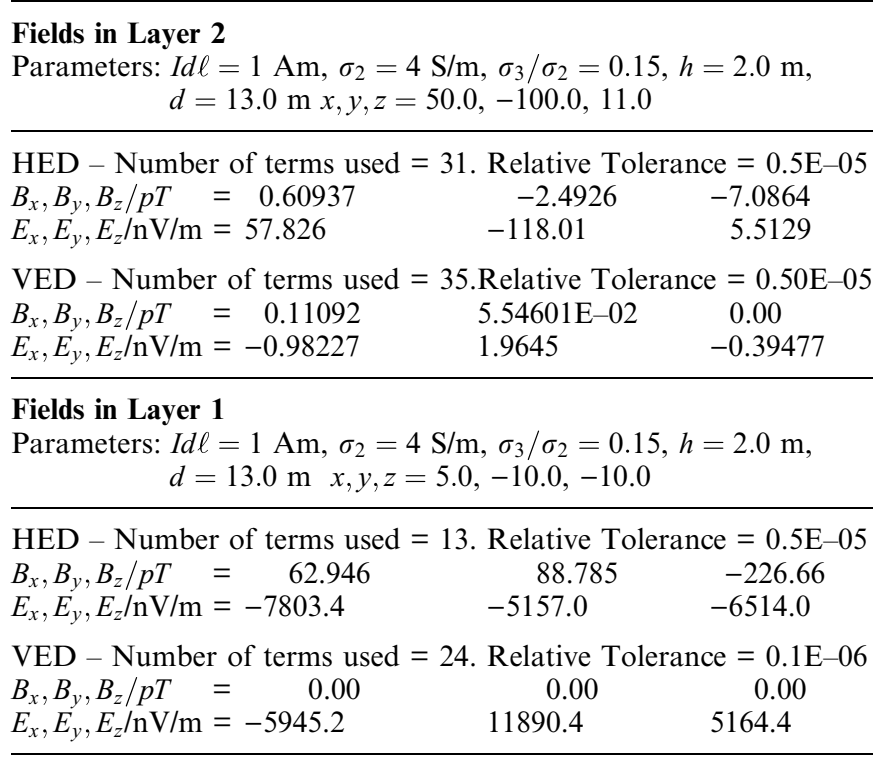

Table 7. Validation of de model against the ac model (layer 1)

\begin{tabular}{lclll}
\hline $\begin{array}{l}\text { Source } \\
\text { Field }\end{array}$ & HED & \multicolumn{3}{l}{ VED } \\
Component & dc Model & $\begin{array}{l}\text { ac Model } \\
(3 \mathrm{~Hz})\end{array}$ & dc Model & $\begin{array}{l}\text { ac Model } \\
(3 \mathrm{~Hz})\end{array}$ \\
\hline$B_{x} / \mathrm{nT}$ & $-6.4717 \mathrm{E}-2$ & $6.4716 \mathrm{E}-2$ & 0.0 & $1.4933 \mathrm{E}-11$ \\
$B_{y} / \mathrm{nT}$ & $5.7130 \mathrm{E}-3$ & $5.5987 \mathrm{E}-3$ & 0.0 & $5.9733 \mathrm{E}-12$ \\
$B_{z} / \mathrm{nT}$ & $2.0091 \mathrm{E}-1$ & $2.0088 \mathrm{E}-1$ & 0.0 & 0.0 \\
$E_{x} / \mu \mathrm{V} / \mathrm{m}$ & -5.5653 & 5.5692 & -2.4261 & 2.4264 \\
$E_{y} / \mu \mathrm{V} / \mathrm{m}$ & 4.2892 & 4.2892 & -6.0653 & 6.0660 \\
$E_{z} / \mu \mathrm{V} / \mathrm{m}$ & -3.6831 & 3.6830 & $-6.8996 \mathrm{E}-1$ & $6.9006 \mathrm{E}-1$ \\
\hline
\end{tabular}

Source location $(x, y, h)=(0,0,4)$; Fields computed at $(6,15,-7)$. Layer parameters: $d=10 \mathrm{~m}, \sigma_{2}=4 \mathrm{~S} / \mathrm{m}, \sigma_{3}=1 \mathrm{~S} / \mathrm{m}$. Id $\ell=1 \mathrm{Am}$.

same results as the dc model at sufficiently low frequencies. We have thus made computations using the dc model and the ac model at a frequency of $3 \mathrm{~Hz}$. The results of the dc calculations and the modulus of the ac values for the various components in layer 1 are given in Table 7. The phase of the ac components $(0$ or $\pi)$ also corresponds to the sign of the dc value. The dc and lowfrequency ac fields in layer 2 also agreed numerically.

Acknowledgements. The work described here has been supported, in part, by the U.K. Defence Research Agency, Winfrith Newburgh, UK. Topical Editor D. J. Webb thanks S. Lovell and P. R. Bannister for their help in evaluating this paper.

\section{References}

Abramowitz, M., and I. A. Stegun, Handbook of Mathematical Functions, Dover Publications, New York, 1968.

Bannister, P. R., New Simplified Formulas for ELF Subsurface-toSubsurface Propagation, IEEE J. Ocean. Eng., OE-9, 154-163, 1984.

Burke, C. P., and D. Ll. Jones, Electromagnetic Wave Propagation in Three-Layered Media, Technical Report RP921015, 1992.

Burke, C. P., and D. Ll. Jones, ELF Propagation in Deep and Shallow Sea Water, AGARD Conf. Proc. 529, AGARD (NATO), Neuilly sur Seine, France, pp 11.1-11.8, 1993.

Burke, C. P., and D. Ll. Jones, A Signal-to-Noise Model for ELF Propagation in Sub-Surface Regions, IEEE J. Ocean. Eng., 19, 353-359, 1994.

Chave, A. D., and C. S. Cox, Controlled Electromagnetic Sources for Measuring Electrical Conductivity Beneath the Oceans, $J$. Geophys. Res., 87, 5327-5338, 1982.

Fraser-Smith, A. C., A. S. Inan, O. G. Villard and R. G Joiner, Seabed Propagation of ULF/ELF Electromagnetic Fields from Harmonic Dipole Sources Located on the Seafloor, Radio Sci. 23, 931-943, 1988.

Kraichman, M. B., Handbook of Electromagnetic Propagation in Conducting Media, U.S. Government Printing Office, Washington, DC 20402, Stock No. 008-040-00074-5, 1976.

Molchanov, O. A., Yu. A Kopytenko, P. V. Voronov, E. A. Kopytenko, T. Matiashvili, A. C. Fraser-Smith, and A. Bernardi, Results of ULF Magnetic Field Emission Near the Epicenter of Spitac $(\mathrm{Ms}=6.9)$ and Loma Prieta $(\mathrm{Ms}=7.1)$ Earthquake, A Comparative Analysis, Geophys. Res. Lett., 19, 1495-1498, 1992.

Parrot, M., A. C. Fraser-Smith, O. A. Molchanov, and T. Yoshino, Electromagnetic effects associated with earthquakes and volcanic eruptions, EOS Trans. Am. Geophys. Union, 76, 233, 1995.

Sommerfeld, A., Partial Differential Equations in Physics, Academic Press, London, New York, 1967. 
Stratton, J. A., Electromagnetic Theory, McGraw-Hill, New York, 1941.

Wait, J. R., Geo-Electromagnetism, Academic Press, London, New York, 1982.

Wait, J. R., Electromagnetic Wave Theory, John Wiley, New York 1987.
Wait J. R., Basic Radiation Fields, Radiosci., 4 (No. 4), 90-94, 1993.

Weaver, J. T., The Quasi-static Field of an Electric Dipole Embedded in a Two Layer Conducting Half Space, Can J. Phys., 45, 1981-2002, 1967. 